\title{
Development of a Clamping Device for Tensile Testing of Intracranial Flow Diverter Stents
}

\author{
Csongor BUKOR, ${ }^{1}$ Dóra KÁROLY, ${ }^{2}$ Benjamin CSIPPA ${ }^{3}$ \\ ${ }^{1}$ Budapest University of Technology and Economics, Faculty of Mechanical Engineering, \\ Department of Materials Science and Engineering, Budapest, Hungary, bukorcsongor@edu.bme.hu \\ ${ }^{2}$ Budapest University of Technology and Economics, Faculty of Mechanical Engineering, \\ Department of Materials Science and Engineering, Budapest, Hungary, kdora@eik.bme.hu \\ ${ }^{3}$ Budapest University of Technology and Economics, Faculty of Mechanical Engineering, \\ Department of Hydrodynamic Systems, Budapest, Hungary, bcsippa@hds.bme.hu
}

\begin{abstract}
The main danger of a brain aneurysm (a sack-like bulge on the vessel wall) is that in the event of a rupture a severe hemorrhage can occur which may cause death. However, if doctors have tools at their disposal, such as numerical models and simulations for analyzing patient-specific blood vessels, they could use them to decide if a particular treatment is necessary and if so, when. For such models, the different mechanical characteristics of the flow control devices are the input data. Several of these mechanical properties of the devices, such as modulus of elasticity and tensile strength, are determined by tensile testing. In the course of our research, we have developed a clamping device suitable for uniaxial tensile testing of flow diverter stents.
\end{abstract}

Keywords: uniaxial tensile testing, mechanical properties, clamping device.

\section{Introduction}

One of the biggest dangers associated with dilated arteries, the so-called aneurysms, is the rupture of the thinned vessel wall as a result of the bleeding that occurs in patients with complications: approx. $15 \%$ die before reaching the hospita [1].

Treatment of intracranial vasodilatation uses, among other things, reticulated biocompatible devices that exert a flow-modifying effect on the damaged vascular section, thereby reducing the pressure on the vessel wall caused by blood flow, thereby thawing the blood in the aneurysm sac $[2,3]$.

The main hemodynamic effect of the devices is the control and slowing of blood flow in the aneurysm. Thus, by altering the dynamic fluid exchange between the vascular section and the vasodilatation, a hemodynamic intra-aneurysmic environment is created that promotes the development of intragastric thrombosis $[4,5]$.

The reliability of the final result of a simulation is greatly influenced by the accuracy of the input data.
The ideal way to test samples is under real physiological conditions, i.e., in their own environment, in vivo testing. However, due to its invasive nature and ethical considerations, it is not applicable in many cases. Instead, only an in vitro study approaching the real boundary conditions can be considered for the study of the given vascular section [6].

The aim of our research is to provide input data for a mechanical model that can be used to simulate the expansion of flow diverter stents in a specific patient-specific aneurysm model. In the stent model, the struts are replaced by surface springs connected at the intersection of the struts and supplemented by fictitious springs in the radial direction of the model. Mechanical properties are modeled through the two different spring types with different spring constants [7]. One depicts surface mechanics (e.g., longitudinal contraction), while the other is required to model radial expansion. This requires tensile testing of stents and detection of diameter change during testing. 


\section{Materials and methods}

\subsection{Flow diverter stents}

The clamping dimensions of the two devices shown in Figure 1 are shown in Table 1. Device A is made of NiTi alloy and device $\mathrm{B}$ is made of $\mathrm{CoCr}$ alloy. Information is given by the manufacturer on the device packaging regarding the length dimension that a device with a constant diameter model would take up in a vessel section under ideal conditions. The manufacturer's length is usually different from the post-dilation values because manual intervention by the operator can affect the length of the device. The measured diameter values in the free voltage state are greater than the nominal diameter specified by the manufacturer. The NiTi stent shown in Figure 1c) clearly shows a marker strut 15 per longitudinal strut in the weave, which is woven into the structure crosswise under 2 struts - above 2 struts. No such marker strut was observed for the $\mathrm{CoCr}$ stent.

\subsection{Clamping design}

From the point of view of the tensile test, the decisive point outside the device is the appropriate clamping design. In our case, this is given a prominent role as the samples examined are very easily damaged.

The following considerations were taken into account in the design of the clamping device. In particular, it must exert a sufficiently large clamping force to prevent the stent from slipping. This force has to be transmitted to the stent over a small area around the circumference in order to ensure uniform deformation and clear determination of the clamping length, and it had to be ensured that the clamping force deforms the stent as little as possible. In addition, the clamp should have a low weight and allow the stent to be gripped quickly and accurately by hand. Finally, the aim was also for simple and cost-effective manufacturability and availability.

Initially, we used an extremely simple construction. The stent was clamped between two thickwalled silicone tubes and the clamping force was applied using a quick-release bandage. With this solution, stents could only be clamped slowly and inaccurately. This problem lead to the design of the clamping device shown in Figure 2. In the new construction, the stent is pulled onto a clamping rod made of an additive rigid polymer from the inside, and the stent is clamped on the outside between two clamping shafts extending around an axis, an O-ring transmitting the force

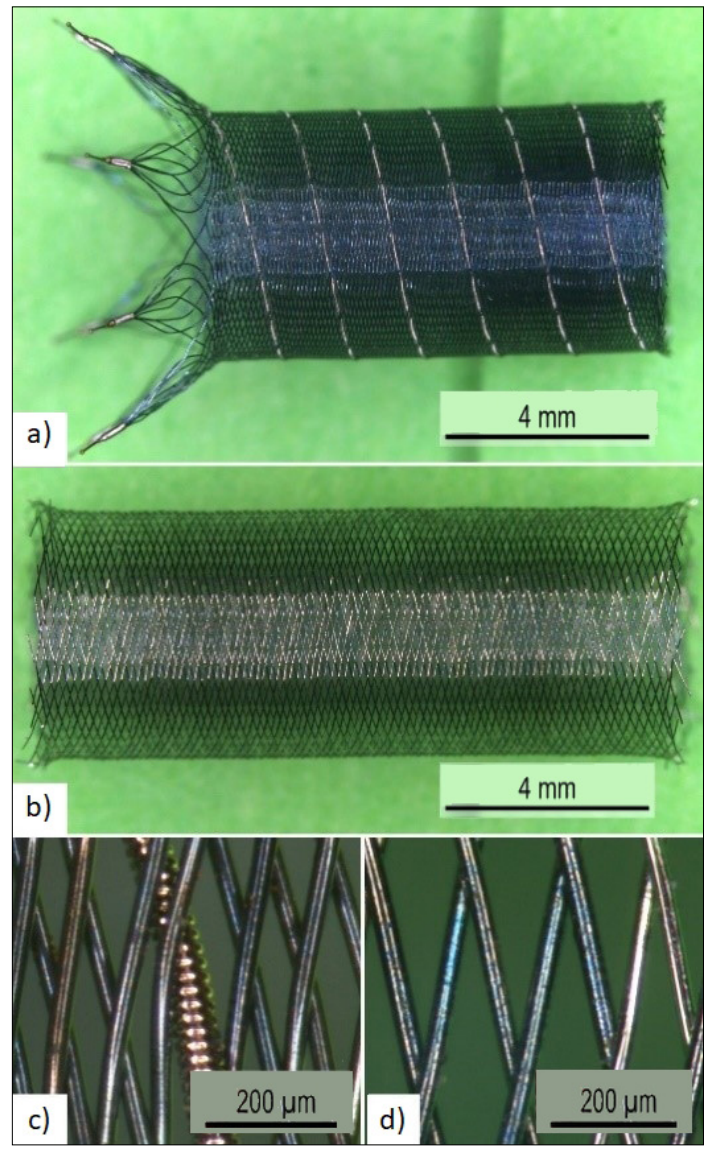

Figure 1. Stereomicroscopic image of Stent $A(a)$, Stent $B(b)$, struts of stent $A(c)$, struts of stent $B(d)$

Table 1. Details of investigated devices.

\begin{tabular}{|l|r|r|}
\hline \multicolumn{1}{|c|}{ Sign } & \multicolumn{1}{c|}{ A } & \multicolumn{1}{c|}{ B } \\
\hline Nominal diameter (mm) & 5 & 5 \\
\hline $\begin{array}{l}\text { Recommended length by manu- } \\
\text { facturer (mm) }\end{array}$ & 24 & 20 \\
\hline Weight (mg) & 25.5 & 29.9 \\
\hline Diameter (mm) & 5.22 & 5.22 \\
\pm 0.019 & \pm 0.034 \\
\hline Length (mm) & 11.63 & 12.75 \\
\pm 0.070 & \pm 0.0391 \\
\hline Material & $\mathrm{NiTi}$ & $\mathrm{CoCr}$ \\
\hline
\end{tabular}

at the clamping point. A groove at the end of the shank allows the clamping force to be applied with a rubber ring of known spring constant. The final clamp was fabricated with Formlabs Form 2 equipment using SLA (stereolithography) additive manufacturing technology. 


\subsection{Measuring method}

Tensile tests were performed using an INSTRON 5965 electromechanical universal material testing device. It is important to note that the device is equipped with a force measuring cell with a measuring range of $\pm 5 \mathrm{~N}$ and an associated small pneumatic clamp, also with a load capacity of $5 \mathrm{~N}$, so that very accurate measurements can be made even in the small force range. The measurement was continued until a $10 \mathrm{~mm}$ probe movement was obtained at a probe speed of $6 \mathrm{~mm} / \mathrm{min}$.

The decrease in diameter during axial pull of the stent was detected with a Nikon D700 camera with a Sigma 70mm 1:2:8:DG macro lens, which was adjusted to take an image at $1 \mathrm{sec}$ intervals from the start of the measurement until the end of the measurement. . This makes the decrease in stent diameter accurately visible and measurable. The diameters were measured halfway between the clamping heads and the diameter change in $\mu \mathrm{m}$ was calculated from this.

\section{Results}

In the study, the curves shown in Figure 3 were obtained, which clearly shows the significant difference between the behavior of the NiTi and $\mathrm{CoCr}$ stents. The NiTi stent, on the other hand, can withstand high forces without diameter change and elongation, and at 50\% elongation can withstand more than twice the force of the $\mathrm{CoCr}$ stent. The initial (vertical) section of the curve shown in Figure 3.b lshows the part where the stents withstood the force without a change in diam-

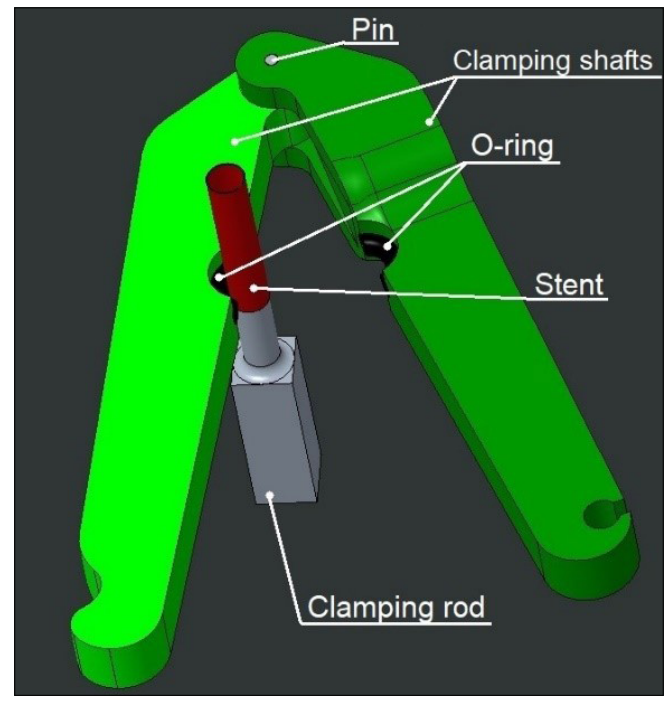

Figure 2. Design of the clamping device. eter, from which the spring constants could be deduced of the surface of the stents in the mechanical model and we could infer the behavior of the radial springs from the closing section of the curve [7].

\section{Conclusions}

The designed clamping device and measurement method is suitable for tensile testing of intracranial flow diverter stents.

As a result of the study, it can be concluded that the NiTi-based stent can withstand a higher force at the same elongation as the CoCr stent.

As a continuation of this research, tensile testing of several different flow diverter devices will be performed with the help of the clamping device and the already mentioned model based on the results will be refined. In addition, the measurement also makes it possible to classify new devices that can also be integrated into the overall mechanical model.

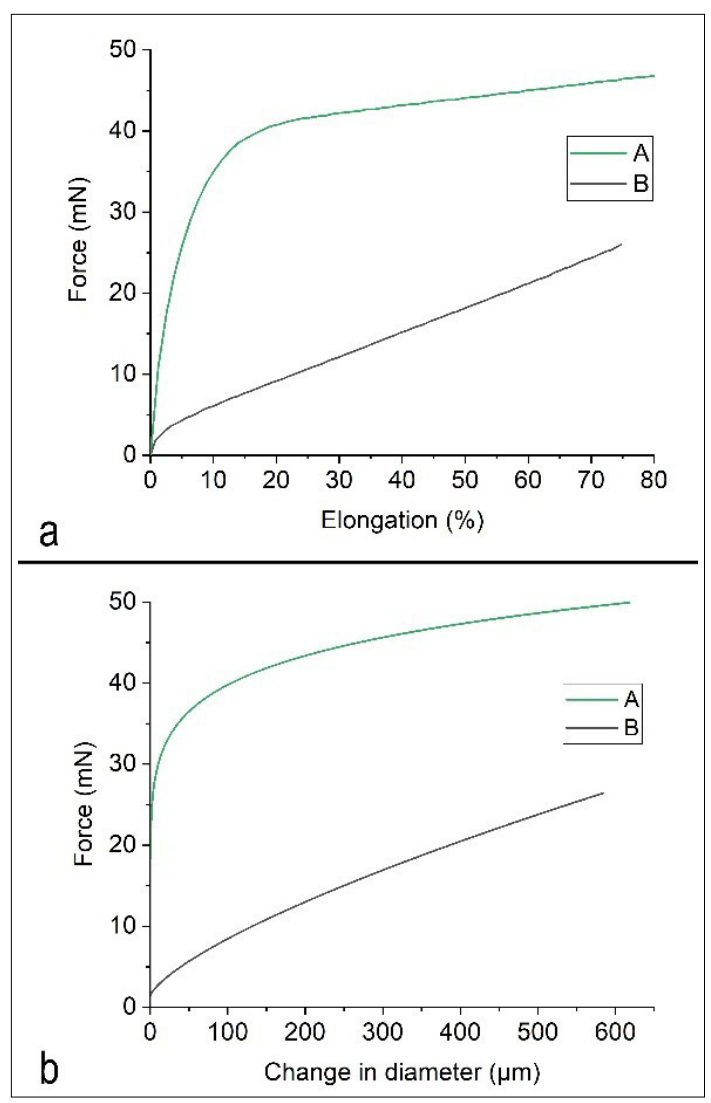

Figure 3. Results of tensile testing of stents $A$ and $B, a$ ) Force-elongation diagram, b) Force-change in diameter diagram 


\section{Acknowledgments}

The publication of the work reported herein has been supported by the NTP-SZKOLL-20-0067 National Talent Programme of the Ministry of Human Capacities and the National Brain Research Program under the contract number 2017-1.2.1-NAP-2017-00002. The research reported in this paper and carried out at BME has been supported by the NRDI Fund (TKP2020 IES, Grant No. BME-IE-NAT; TKP2020 NC, Grant No. BMENCS) based on the charter of bolster issued by the NRDI Office under the auspices of the Ministry for Innovation and Technology.

\section{References}

[1] Chalouhi N., Hoh B.L., Hasan D.: Review of cerebral aneurysm formation, growth and rupture. Stroke, 44/12. (2013) 3613-3622. http://doi.org/10.1161/STROKEAHA.113.002390

[2] Szikora I., Berentei Z., Kulcsar Z., Marosfoi M., Vajda Z. S., Lee W., Berez A., Nelson P. K.: Treatment of intracranial aneurysms by functional reconstruction of the parent artery: the Budapest experience with the pipeline embolization device. American Journal of Neuroradiology, 31/6. (2010) 1139-1147.

http://doi.org/10.3174/ajnr.A2023
[3] Alderazi Y. J., Shastri D., Kass-Hout T., Prestigiacomo C. J., Gandhi C. D.: Flow diverters for intracranial aneurysms. Stroke Research and Treatment, 2014. 1-12.

http://doi.org/10.1155/2014/415653

[4] Asztalos L., Nagy P., Bognár E., Szikora I.: Pattern Change of Flow Diverters due to Bending. Journal of Biomechanical Engineering and Biosciences, 4. (2017) 19-22.

[5] Fu W., Xia Q.: Interaction between flow diverter and parent artery of intracranial aneurysm: a computational study. Applied Bionics and Biomechanics, 1. (2017) 1-9. http://doi.org/10.1155/2017/3751202

[6] Ugron Á., Paál Gy.: On the boundary conditions of cerebral aneurysm simulations. Periodica Polytechnica Mechanical Engineering, 58/1. (2014) $37-45$. http://doi.org/10.3311/PPme.7392

[7] Závodszky G., Csippa B., Paál Gy., Szikora I.: A novel virtual flow diverter implantation method with realistic deployment mechanics and validated force response. International Journal of Numerical Methods in Biomedical Engineering, 36/6. (2020) e3340.

http://doi.org/10.1002/cnm.3340 